INVESTIGACIÓN ORIGINAL

\title{
Infección por HPV de alto riesgo y lesiones intraepiteliales anales en hombres HIV positivos que tienen sexo con hombres
}

\author{
Recibido:12/06/17 - Aceptado: 28/01/18
}

Valeria Irene Fink ${ }^{1}$, Laura Svidler López², Fernanda González ${ }^{3}$, Mariana Tejo을 Gisela Presencia², Gabriela Sidra², María Victoria Galperín $^{2}$, Rita Ofelia L. Pastore², Silvina Figurelli², Lilia Mammana ${ }^{3}$, Carina Cesar ${ }^{1}$, Omar Sued ${ }^{1}$, María Belén Bouzas ${ }^{3}$, Pedro Cahn' ${ }^{1}$.

\section{RESUMEN}

Introducción: El cáncer anal, asociado a la infección con virus de papiloma humano de alto riesgo (HPV-AR), es muy frecuente en hombres que tienen sexo con hombres (HSH) $\mathrm{HIV+.}$

Objetivo: Evaluar frecuencia de infección por HPV-AR, genotipos y lesiones asociadas, y factores asociados.

Materiales y métodos: Estudio en HSH HIV+ (septiembre 2012-marzo 2014, Hospital Fernández). Se recogió información demográfica, de HIV, HPV y prácticas sexuales. Se realizó citología anal, detección de HPV-AR (HC2 HighRisk HPV DNA, Digene ${ }^{\circledR}$ ) y genotipificación en las muestras

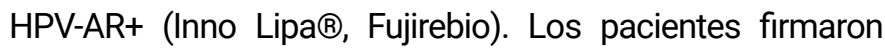
consentimiento informado. Se indicó tratamiento según resultados.

Resultados: Completaron el estudio 57 pacientes. Mediana de edad: 40 años (rango intercuartil [RIC]: 29-45); de CD4: 444 cels/mm3 (RIC: $345-568$ ); 77\% recibían tratamiento antirretroviral, $68 \%$ con carga viral no detectable. Citologías: negativas (24\%); lesión intraepitelial de bajo grado (54\%); lesión intraepitelial de alto grado (20\%); ASCUS (2\%). El $80 \%$ fue HPV-AR+. Los pacientes con diagnóstico de HPV-AR $(p=0,006)$ y de lesión intraepitelial tuvieron CD4 $<500$ cels/ mm3 con más frecuencia $(p=0,030)$. Los pacientes con
${ }^{1}$ Fundación Huésped, Buenos Aires, Argentina ${ }^{2}$ Hospital J. A. Fernández, Buenos Aires, Argentina

${ }^{3}$ Hospital J.F. Muñiz, Buenos Aires, Argentina

Dirección de correspondencia: Dra. Valeria Fink, Pasaje Carlos Gianantonio 3932, Buenos Aires, CP 1202, Argentina. valeria.fink@huesped.org.ar

Ninguno de los autores declara presentar conflicto de intereses en relación a esta publicación.

Financiación parcial: Instituto Nacional del Cáncer, Ministerio de Salud de Nación.

Los contenidos del material a ser evaluado no han sido publicados anteriormente. El trabajo se presentó en el XV Congreso Argentino de la Sociedad Argentina de Infectología (2015). 
HPV-AR tuvieron mayor frecuencia de carga viral detectable $(p=0,020$, prueba de Fisher). El porcentaje de pacientes con uso consistente de preservativo fue mayor entre los pacientes sin lesión citológica $(p=0,026)$. Genotipos de alto riesgo más frecuentes: HPV-16 (51\%), HPV-31 (44\%) y HPV-51 (40\%); de bajo riesgo: HPV-6 (47\%) y HPV-44 (35\%).

Conclusiones: Se encontró elevada frecuencia de lesión citológica (76\%) y de HPV-AR (80\%). Es necesario establecer estrategias de prevención en esta población incluyendo tamizaje, vacunación y promoción de sexo seguro.

Palabras clave: HIV, lesión intraepitelial anal, HPV, citología anal, tamizaje de cáncer anal, hombres que tienen sexo con hombres.

\section{Introducción}

La infección por el virus del papiloma humano (HPV) constituye la enfermedad de transmisión sexual más frecuente, estimándose que un $80 \%$ de las personas sexualmente activas van a adquirir esta infección en algún momento de su vida (1). Existen aproximadamente 40 genotipos de HPV que afectan la zona anogenital y de éstos unos 15 son considerados oncogénicos y se conocen como de alto riesgo (HPV-AR) (2). Varios cánceres anogenitales así como de cabeza y cuello se han visto asociados con la presencia de estos genotipos $(1,3,4)$.

El carcinoma anal de células escamosas (CAE) es un tumo poco frecuente en la población general, aunque su incidencia estimada ha ido aumentando un $2,2 \%$ anual en los últimos diez años (5). Se estima que el $80-90 \%$ de estos tumores están asociados con la presencia de HPV de alto riesgo oncogénico. Se trata de uno de los cánceres no definitorios de sida que afecta a la población HIV+ con una frecuencia notoriamente mayor que a la población HIV negativa (ies 20-130 veces mayor) (6-8). Su incidencia ha aumentado desde el advenimiento del tratamiento antirretroviral de alta eficacia, posiblemente relacionado con la mayor sobrevida tumores (9-11).

La inmunosupresión es un factor muy importante en el desarrollo de lesiones asociadas al HPV, incluyendo tumores malignos como el de cuello uterino y de ano. La inmunidad celular es clave en la eliminación del HPV. Al encontrarse alterada en la infección por HIV, las personas HIV+ tienen mayor prevalencia de HPV, mayor riesgo de presentar infecciones persistentes, con múltiples genotipos, de presentar lesiones preneoplásicas y de progresar a cáncer $(12,13)$.

La población de hombres que tiene sexo con hombres (HSH), en especial aquellos con infección por HIV, es de las más afectadas por este cáncer, siendo el cuarto cáncer más prevalente en este grupo $(9,14)$. Por otra parte, las lesiones anales intraepiteliales de alto grado en HSH HIV+ tienen mayor potencial de progresar a cáncer anal (15). Al igual que el cáncer de cuello uterino, el cáncer anal y sus lesiones precursoras pueden de la citologia y la anoscopía de alta resolución (AAR), aunque aún no existe consenso sobre la metodología más costoefectiva para tamizaje de cáncer anal.

En Argentina, la información sobre prevalencia de HPV y en la población HIV positiva. Un estudio realizado en la ciudad de Buenos Aires evidenció un $83,5 \%$ de prevalencia de HPV anal en HSH (16). Según la Agencia Internacional de Investigación en Cáncer (IARC), la frecuencia de cáncer anal en los centros centinela de Argentina fue entre 0,1 y 0,3\% en hombres y 0-0,6 $\%$ en mujeres en el período 2003-2007 (17).

El presente estudio evaluó la presencia de HPV-AR y de lesiones asociadas a HPV en hombres que tienen sexo con hombres HIV+ y su asociación con edad, prácticas sexuales, consumo de tabaco y variables relacionadas a la infección por HIV.

\section{Materiales y métodos}

Diseño

Se realizó un estudio transversal desde septiembre 2012 a marzo 2014. Se evaluó la prevalencia de HPV-AR así como la prevalencia de lesiones citológicas y la asociación entre la presencia de HPV-AR y las características demográficas, de hábitos y relacionadas con HIV.

Población

Los pacientes HSH HIV+ fueron invitados a participar en el estudio durante su consulta de rutina. El reclutamiento de pacientes se realizó en la División Infectología del Hospital Juan A. Fernández, Ciudad de Buenos Aires. Se les explicó el estudio y se obtuvo consentimiento informado por escrito de todos los participantes. El estudio fue aprobado por el Comité de Ética en Investigación del Hospital Fernández y el Comité de Ética en Investigación de Fundación Huésped.
Los principales criterios de elegibilidad fueron: HSH (según autodefinición de los sujetos), edad $\geq 18$ años, infección confirmada por HIV (pruebas de tamizaje y confirmación positivas y/o una carga viral >1000 copias/mm3).

\section{Procedimientos del estudio}

Se completó una ficha estructurada para cada participante. Se diseñó un cuestionario incluyendo información demográfica historia de la infección por HIV, hábitos, prácticas sexuales y frecuencia de relaciones sexuales. La ficha se completó con información provista por el paciente asícomo la proven de su historia clínica. Se consideran valos de estudios de linfocitos T CD4 y carga viral (CV) de HIV que se hubieran realizado dentro de los seis meses de la fecha del estudio. Se consideró uso consistente de preservativo el uso en todas las prácticas con penetración.

A todos participantes se les realizó un examen proctológico donde se tomó una muestra para citología anal y una para detección de HPV-AR con Digene cervical sampler® y se realizó anoscopía de alta resolución.

Las citologías se clasificaron según el sistema de Bethesda siendo negativas (sin patologí oncológica), escamosas atípicas de significado indeterminado, (atypical squamous cells of undetermined significance, ASC-US), ASC-H, (atypical squamous cells), lesiones intraepiteliales escamosas de bajo grado (LIE-BG), lesiones intraepiteliales escamosas de alto grado (LIE-AG) y carcinoma de células escamosas (18). La detección de HPV-AR se realizo Hybrid Capture 2 HightRisk HPV DNA Test"w-Digen prueba es un ensayo de hibridización de ácidos nucleicos que permite detectar en forma cualitativa 13 genotipos de HPV-AR (HPV-16/18/31/33/35/39/45/51/52/56/58/59/68). En las muestras que resultaron positivas para HPV-AR se realizó la detección de genotipos a través de Inno-Lipa HPV Genotyping Extra II, Fujirebio. Esta es una prueba de hibridización reversa previa amplificación de la muestra por PCR que permite identificar 28 genotipos de HPV (HPV 16/18/26/31/33/35/39/45/51/52/53/56/58/59/66/68/82, de alto riesgo; HPV-6/11/40/43/44/54/70, de bajo riesgo; HPV-69/71/ 73/ 74, de riesgo desconocido) (19).

Los resultados de los estudios se ingresaron en una base de datos junto con la información obtenida en la ficha. Los pacientes recibieron seguimiento y tratamiento de acuerdo con los resultados.

Análisis estadístico

Las siguientes variables se estudiaron como variables Se observó asociación estadísticamente significativa entre continuas: edad, edad a la primera relación sexual, número de parejas sexuales a lo largo de la vida, tiempo entre el diagnóstico de HIV y el momento del estudio y los CD4. Las variables: uso consistente de preservativo y consumo de tabaco alguna vez en la vida se consideraron en forma categórica. Asimismo, a efectos del análisis, las siguientes variables se organizaron en forma dicotómica: se estratificaron los CD4 en mayores y menores de 500 células/ $\mathrm{mm} 3$, las cargas virales de HIV en detectable y no detectable, presencia o ausencia de lesiones asociadas a HPV, definida como la presencia de cualquier lesión asociada a HPV en la citología y la presencia o ausencia de lesiones intraepiteliales de alto grado. Para evaluar la asociación entre las variables estudiadas y la prevalencia de HPV-AR y lesiones citológicas se utilizó regresión logística bivariada. Cuando no fue posible realizar regresión logística por la distribución de los casos (ningún caso sin lesión tenía carga viral detectabe) se utilizó la prueba de Fisher Se utilizó IBM SPSS Statistics 20.

\section{Resultados}

Se presentan los datos de los 57 sujetos que completaron estudio. La mediana de edad fue de 40 años (rango intercuartil, RIC: 29-45). La edad al inicio de su vida sexual fue de 16 años (RIC: 14-19). La mediana de número de parejas sexuales autorreferida a lo largo de la vida fue 20 (RIC: $10-$ 20). Un $56 \%$ refirió usar preservativo en forma consistente. El tiempo de infección con HIV fue de una mediana de 6 años (RIC: 3 -10) A momento del estudio (+/-6 meses) la median tratamiento ARV y el $68 \%$ presentaba $C V$ no detectable.

Los resultados de la citología fueron: $24 \%$ sin patología oncologica, 54\% lesión intraepitelial de bajo grado (LIE-BG) $2 \%$ células escamosas anómalas de significado incierto (ASCUS, por su nombre en inglés atypical squamous cells of undetermined significance), $20 \%$ lesión intraepitelial de alto grado (LIE-AG). La AAR fue anormal en $63 \%$ de las citologías negativas, $65 \%$ de LIE-BG y $80 \%$ de LIE-AG.

De los 57 pacientes, se obtuvo muestra para detección de HPV-AR on 55 (en uno no pudo realizarse por intolerancia a procedimiento y en otro no se pudo amplificar la muestra). El $80 \%(44 / 55)$ de estas muestras fueron positivas para HPVAR (incluyendo todos los LIE-AG y el $83 \%-24 / 29$ - de los LIE-BG). La Tabla 1 muestra los resultados de los diferentes diagnosticos citológicos según la presencia de HPV-AR.

Se observó asociación estadísticamente significativa entre 
la presencia de HPV-AR y el valor de CD4. La mediana del los sujetos sin lesiones citológicas $(85 \%$ vs $46 \%)$, $(\mathrm{p}=0,026)$. recuento de linfocitos CD4 en los pacientes con HPV-AR fue No se observaron otras asociaciones significativas (Tabla 3). significativamente menor que en los pacientes negativos

( 473 cels $/ \mathrm{mm} 3$ vs 657 cels $/ \mathrm{mm} 3, \mathrm{p}=0,035$ ). Por otro lado, se Se estudiaron los genotipos en 43 de las 44 muestras con observóunamayor frecuencia de pacientes con peorsituación HPV-AR.

inmunológica (CD4 <500 cels/mm3) entre los pacientes con

HPV-AR que en los negativos (76\% vs $27 \%, p=0,006)$. Se Entre los genotipos de alto riesgo, los más frecuentes fueron encontró asimismo que todos los pacientes negativos para el HPV-16 ( $n=22), \mathrm{HPV}-31 \quad(n=19), \mathrm{HPV}-51 \quad(n=17), \mathrm{HPV}-18$ HPV-AR (11/11) tuvieron CV no detectable, mientras que en $\quad(n=11)$, HPV-66 $(n=16)$ y HPV-53 $(n=14)$. Entre los de bajo aquellos en quienes se había detectado HPV-AR, la CV fue no riesgo, el HPV-6 fue el más frecuente ( $n=20), H P V-44(n=15)$ y detectable en el $60 \%$ de los casos $(21 / 35)(p=0,020$, prueba HPV-11 ( $n=9)$ (Figura 1$)$.

de Fisher) (Tabla 2)

Los pacientes con lesión citológica anal presentaron mayor frecuencia de CD4 $<500$ cels $/ \mathrm{mm} 3$ que aquellos sin lesión

citológica $(73 \%$ vs $36 \%)$, $(\mathrm{p}=0,030)$. Se observó también

mayor frecuencia de uso consistente de preservativo entre

\section{Tabla 1. Resultados de las citologías en muestras positivas}

y negativas para HPV-AR

\begin{tabular}{l|c|c|}
\multicolumn{1}{c|}{$\begin{array}{c}\text { Resultados }\left(n=55^{*}\right) \\
\text { Citologia }\end{array}$} & $\begin{array}{c}\text { HPV-AR negativa } \\
(n=11,20 \%)\end{array}$ & $\begin{array}{c}\text { HPV-AR positiva } \\
\left(n=44^{*}, 80 \%\right)\end{array}$ \\
Negativo $(n=13,24 \%)$ & 5 & 8 \\
$\begin{array}{l}\text { Lesión intraepitelial } \\
\text { de bajo grado } \\
\left(n=30^{*}, 54 \%\right)\end{array}$ & 5 & 24 \\
$\begin{array}{l}\text { Lesión intraepitelial } \\
\text { de alto grado } \\
(n=11,20 \%)\end{array}$ & 0 & 11 \\
ASCUS $(n=1,2 \%)$ & 1 & 0 \\
&
\end{tabular}

*1 tenía citología no representativa

\# 1 no se pudo procesar la prueba de HPV-AR

\section{Aclaraciones:}

HPV-AR: Virus de Papiloma Humano de alto riesgo oncogénico

ASCUS: del inglés atypical squamous cells of undetermined significance
Tabla 2. Análisis de las variables estudiadas en relación a la presencia de HPV-AR

\begin{tabular}{|c|c|c|c|c|}
\hline Características & $\begin{array}{l}\text { HPV-AR negativo } \\
(n=11)\end{array}$ & $\begin{array}{l}\text { HPV-AR positivo } \\
(n=44)\end{array}$ & OR & $\mathbf{p}$ \\
\hline $\begin{array}{l}\text { Edad (mediana-RIC) } \\
\text { (años) }(n=55)\end{array}$ & $42(29-46)$ & $44(38-45)$ & $\begin{array}{c}0,986 \\
(0,917-1,061)\end{array}$ & 0,713 \\
\hline \multicolumn{5}{|l|}{$\begin{array}{l}\text { Prácticas sexuales } \\
\text { y hábitos }\end{array}$} \\
\hline $\begin{array}{l}\text { Uso consistente de } \\
\text { preservativo }(n-\%) \\
(n=52)\end{array}$ & $\begin{array}{l}8 / 11 \\
(73 \%)\end{array}$ & $\begin{array}{l}22 / 41 \\
(54 \%)\end{array}$ & $\begin{array}{c}2,303 \\
(0,534-9,937)\end{array}$ & 0,263 \\
\hline $\begin{array}{l}\text { Edad a la primera } \\
\text { relación sexual } \\
\text { (mediana-RIC) (años) } \\
\text { (n=52) }\end{array}$ & $18(15-20)$ & $16(14-19)$ & $\begin{array}{c}0,918 \\
(0,78-1,080)\end{array}$ & 0,302 \\
\hline $\begin{array}{l}\text { Número de parejas } \\
\text { sexuales a lo largo de } \\
\text { la vida (mediana-RIC) } \\
\text { ( } \mathrm{n}=52)\end{array}$ & $12(7-50)$ & $20(10-62)$ & $\begin{array}{c}1,011 \\
(0,989-1,033)\end{array}$ & 0,328 \\
\hline $\begin{array}{l}\text { Consumo de tabaco } \\
\text { alguna vez en la vida } \\
(n-\%)(n=55)\end{array}$ & $\begin{array}{l}6 / 11 \\
(55 \%)\end{array}$ & $\begin{array}{l}28 / 44 \\
(64 \%)\end{array}$ & $\begin{array}{c}1,458 \\
(0,383-5,549)\end{array}$ & 0,580 \\
\hline \multicolumn{5}{|l|}{$\begin{array}{l}\text { Variables relacionadas } \\
\text { con HIV }\end{array}$} \\
\hline $\begin{array}{l}\text { Tiempo entre el } \\
\text { diagnóstico de HIV y el } \\
\text { estudio (mediana-RIC) } \\
\text { (años) (n=54) }\end{array}$ & $8(4-10)$ & $5(2-10)$ & $\begin{array}{c}0,961 \\
(0,86-1,07)\end{array}$ & 0,472 \\
\hline $\begin{array}{l}\text { Recuento de CD4 } \\
\text { (mediana- RIC) (cels/ } \\
\text { mm3) }(n=52)\end{array}$ & $\begin{array}{c}657 \\
(451-756)\end{array}$ & $\begin{array}{c}413 \\
(282-502)\end{array}$ & $\begin{array}{c}0,997 \\
(0,995-1)\end{array}$ & 0,035 \\
\hline $\begin{array}{l}\text { Pacientes con CD4< } \\
500 \text { cels } / \mathrm{mm} 3(n=52)\end{array}$ & $\begin{array}{l}3 / 11 \\
(27 \%)\end{array}$ & $\begin{array}{l}31 / 41 \\
(76 \%)\end{array}$ & $\begin{array}{c}8,267 \\
(1,833-37,280)\end{array}$ & 0,006 \\
\hline $\begin{array}{l}\text { Carga viral < } 50 \text { copias/ } \\
\text { mm3 (pacientes } \\
\text { en tratamiento } \\
\text { antiretroviral) } \\
(\mathrm{n}-\%) *(\mathrm{n}=46)\end{array}$ & $\begin{array}{l}11 / 11 \\
(100 \%)\end{array}$ & $\begin{array}{l}21 / 35 \\
(60 \%)\end{array}$ & & 0,020 \\
\hline
\end{tabular}

* Prueba de Fisher

OR: Odds ratio

IC 95\%: Intervalo de confianza 95\% 


\section{Tabla 3. Análisis de las variables estudiadas en relación a la presencia de lesión}

\begin{tabular}{|c|c|c|c|c|}
\hline Características & $\begin{array}{c}\text { Ausencia de } \\
\text { lesión citológica } \\
(n=13)\end{array}$ & $\begin{array}{l}\text { Presencia de } \\
\text { lesión citológica } \\
(n=42)\end{array}$ & OR IC $95 \%$ & $\mathbf{p}$ \\
\hline $\begin{array}{l}\text { Edad (mediana- RIC) } \\
\text { (años) (n=55) }\end{array}$ & $35(28-44)$ & $40(32-45)$ & $\begin{array}{c}1,037 \\
(0,964-1,116)\end{array}$ & 0,329 \\
\hline \multicolumn{5}{|l|}{$\begin{array}{l}\text { Conducta sexual } \\
\text { y hábitos }\end{array}$} \\
\hline $\begin{array}{l}\text { Uso consistente de } \\
\text { preservativo }(\mathrm{n}-\%) \\
(\mathrm{n}=52)\end{array}$ & $\begin{array}{l}11 / 13 \\
(85 \%)\end{array}$ & $\begin{array}{l}18 / 39 \\
(46 \%)\end{array}$ & $6,417(1,254-32,844)$ & 0,026 \\
\hline $\begin{array}{l}\text { Edad a la primera } \\
\text { relación sexual } \\
\text { (mediana- RIC) (años) } \\
\text { (n=52) }\end{array}$ & $17(15-19)$ & $16(14-19)$ & $\begin{array}{c}1,028 \\
(0,892-1,185)\end{array}$ & 0,703 \\
\hline $\begin{array}{l}\text { Número de parejas } \\
\text { sexuales a lo largo de } \\
\text { la vida (mediana-RIC) } \\
(\mathrm{n}=52)\end{array}$ & $10(6-20)$ & $20(10-50)$ & $\begin{array}{c}1,013 \\
(0,990-1,037)\end{array}$ & 0,261 \\
\hline $\begin{array}{l}\text { Consumo de tabaco } \\
\text { alguna vez en la vida } \\
(n-\%)(n=55)\end{array}$ & $\begin{array}{l}9 / 13 \\
(69 \%)\end{array}$ & $\begin{array}{l}26 / 42 \\
(62 \%)\end{array}$ & $\begin{array}{c}0,722 \\
(0,191-2,737)\end{array}$ & 0,632 \\
\hline \multicolumn{5}{|l|}{$\begin{array}{l}\text { Variables relacionadas } \\
\text { al HIV }\end{array}$} \\
\hline $\begin{array}{l}\text { Tiempo entre el } \\
\text { diagnóstico de HIV y el } \\
\text { estudio (mediana-RIC) } \\
\text { (años) (n=54) }\end{array}$ & $4(1-9)$ & $7(4-14)$ & $\begin{array}{c}0,961 \\
(0,86-1,07)\end{array}$ & 0,05 \\
\hline $\begin{array}{l}\text { Recuento de CD4 } \\
\text { (mediana RIC) (cels/ } \\
\text { mm3) }(n=52)\end{array}$ & $\begin{array}{c}657 \\
(343-548)\end{array}$ & $\begin{array}{c}441 \\
(338-540)\end{array}$ & $\begin{array}{c}0,997 \\
(0,995-1)\end{array}$ & 0,127 \\
\hline $\begin{array}{l}\text { Pacientes con CD4 < } \\
500 \text { cels/mm3 }(n-\%) \\
(\mathrm{n}=52)\end{array}$ & $\begin{array}{l}4 / 11 \\
(36 \%)\end{array}$ & $\begin{array}{l}30 / 41 \\
(73 \%)\end{array}$ & $\begin{array}{c}8,267 \\
(1,833-37,280)\end{array}$ & 0,030 \\
\hline $\begin{array}{l}\text { Carga viral }<50 \\
\text { copias } / \mathrm{mm} 3 \text { entre } \\
\text { los pacientes } \\
\text { en tratamiento } \\
\text { antirretroviral } \\
(\%) *(n=45))\end{array}$ & $\begin{array}{c}8 / 9 \\
(89 \%)\end{array}$ & $\begin{array}{l}24 / 36 \\
(67 \%)\end{array}$ & $\begin{array}{c}4 \\
(0,447-35,788)\end{array}$ & 0,215 \\
\hline
\end{tabular}

OR: Odds ratio

IC 95\%: Intervalo de confianza 95\%
Figura 1. Genotipos de HPV en hombres $(n=43)$

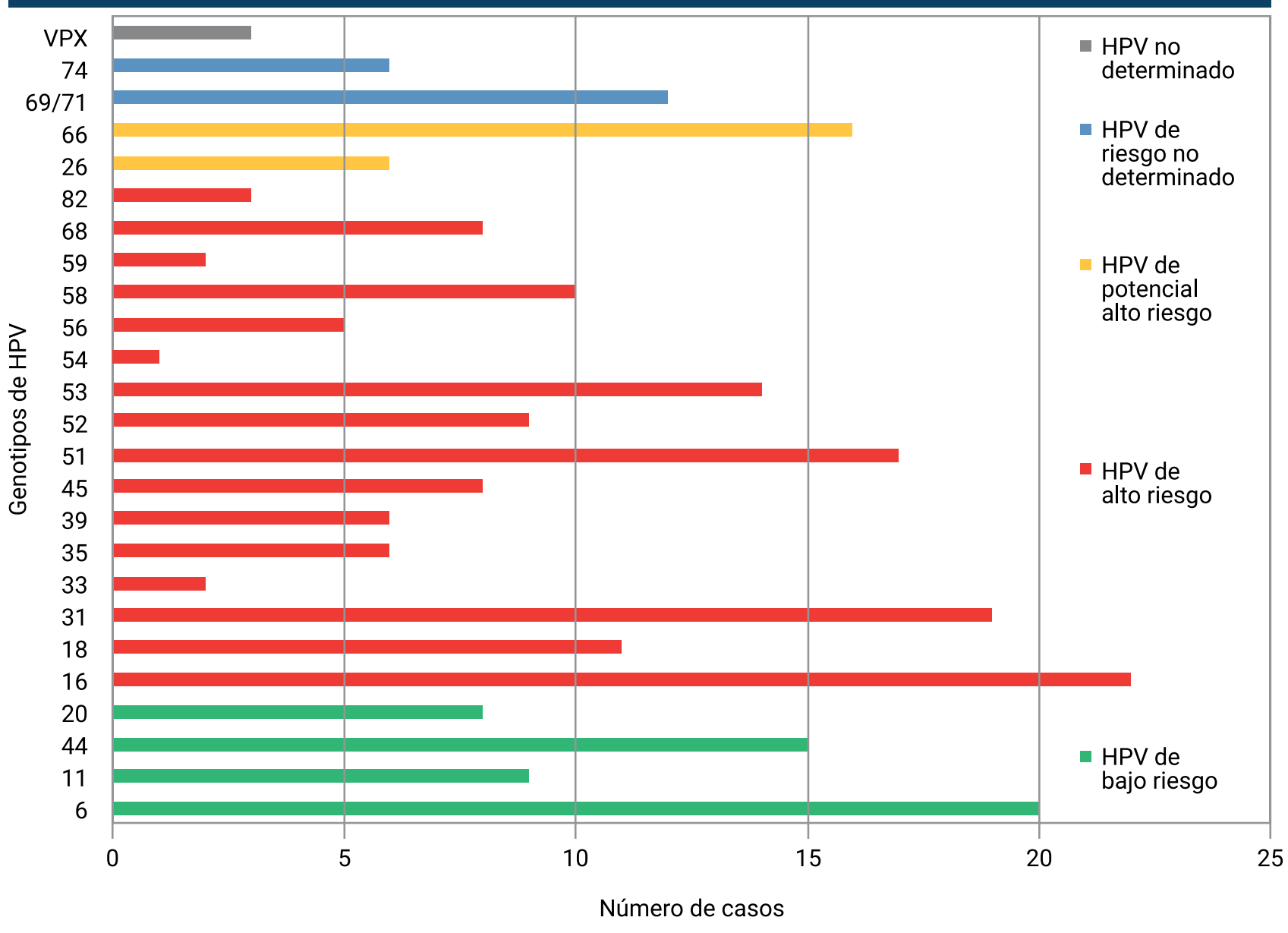




\section{Discusión}

$\mathrm{CD} 4<50 \mathrm{cel} / \mathrm{mm} 3$ e infecciones por múltiples genotipos $(28)$ (2) 列 por HIV. Se diagnosticó además la presencia de lesiones estudios que han reportado información contradictoria en intraepiteliales en el $76 \%$ de los pacientes $(20 \%$ de alto relación a la prevención de la transmisión de HPV con el grado y $54 \%$ de bajo grado). Todos los pacientes con LIE-AG uso de preservativo $(29,30)$. Es posible que un estudio con presentaron HPV-AR. La mediana de CD4 al momento del mayor número de pacientes permita identificar mejor estos estudio fue de $444 \mathrm{cels} / \mathrm{mm} 3$, por lo cual se trató de pacientes factores. Nuestros hallazgos confirman que esta patología sin severo deterioro inmunológico o que ya lo habían puede afectar a todos los pacientes HSH con infección mejorado luego del inicio de tratamiento antirretroviral. Es por HIV independientemente del tiempo de evolución de la destacable la persistencia de conductas de riesgo, dado que infección y del uso de tratamiento antirretroviral, aunque una solo aproximadamente la mitad de los pacientes refirieron el mejor situación inmunológica parece relacionarse con la uso consistente de preservativo.

La elevada prevalencia de HPV-AR encontrada en En relación alos genotipos, otras publicaciones han reportado (20) elevada frecuencia de HPV-16 (22\%-28\%) aunque menor a la internacionales (20-24) como locales (16). Es importante hallada en este estudio (51\%). El genotipo HPV-51 también recordar que el grupo de HSH viviendo con HIV se encuentra fue frecuente así como el HPV-52 $(23,24)$. La distribución entre los de mayor frecuencia de HPV-AR en región anal de genotipos de otro estudio realizado en nuestro país en $(48,9-94,4 \%)(20,15)$. Se estima que la prevalencia en mujeres hombres que tienen sexo con hombres fue diferente $(16)$. HIV positivas es de $16-85 \%$, en mujeres HIV negativas es Se debe considerar que la evaluación de HPV en cada de 4-86\% (26), en hombres heterosexuales HIV+ es de 42- estudio fue hecha con diferentes métodos. El HPV-16 fue el $46 \%$ (13) y en hombres que tienen sexo con hombres HIV más frecuente genotipo de alto riesgo, afectando al $51 \%$ de negativos de $37,2-45 \%(20,24,25)$ evidenciando el riesgo que los pacientes del presente estudio y el $24,5 \%$ en el estudio presenta esta población de desarrollar lesiones asociadas a de Pando et al. Los genotipos de alto riesgo que siguieron HPV incluyendo cáncer anal.

En relación a las lesiones anales diagnosticadas por citologia, en el $76 \%$ de los casos se ha encontrado algún tipo de patología y en un $20 \%$ lesiones de alto grado. En este estudio no se detectó ningún caso de cáncer. Un meta análisis que comprende estudios publicados hasta noviembre de 201 encontró una prevalencia global de lesiones de $55,1 \%$ en $\mathrm{HSH}$ HIV+ y de $29,2 \%$ en HSH HIV- mientras que la prevalencia de lesiones de alto grado fue $23,9 \%$ y $15,2 \%$ respectivamente (20).

Los factores asociados en forma significativa con la presencia de HPV-AR fueron el valor de CD4 al momento del estudio y la carga viral. Los niveles de CD4 $<500$ cels/ $\mathrm{mm} 3$ se asociaron con la presencia de lesiones. No hemos encontrado asociación con otros factores descriptos en otras publicaciones, como el número de parejas sexuales, la edad de inicio de relaciones sexuales o el uso de tabaco. Un estudio de la cohorte suiza encontró asociación entre el uso de tabaco, la presencia de HPV con un bajo de recuento de CD4 y el desarrollo de cáncer anal (27). Otro estudio de los Estados Unidos evidenció asociación entre la aparición de lesiones y un recuento de $\mathrm{CD} 4<500 \mathrm{cel} / \mathrm{mm} 3$, un nadir (en personas $(8,9 \%)$, HPV-66 (10,4\%) y HPV-33 (7,2\%). Las frecuencias de infección fueron mucho más elevadas en el estudio que se presenta y algunos genotipos como HPV-51 (40\%) fueron muy frecuentes. Entre los genotipos de bajo riesgo, en ambos HPV-6 fue el más frecuente, siendo más bajo en el estudio de Pando (28,6\% vs $46 \%)$. Existen varias posibles explicaciones para estas diferencias. El estudio de Pando incluyó HSH HIV+ y HIV-, con una prevalencia de infección por HIV del 17,3\%, mientras que este estudio incluye sólo pacientes HIV+. Desconocemos si puede haber diferencias por ser distintos años de evaluación y el distinto lugar de reclutamiento.

Si bien en este estudio hay pacientes que por edad quedarían fuera de la indicación de vacuna, más de la mitad de los pacientes se beneficiaría de recibirla y la otra mitad se hubiera be genotipo se realizó sólo en aquéllas muestras que tuvieron resultado positivo para HPV-AR, por lo cual el número de potenciales beneficiados podría ser aún mayor.

Entre las limitaciones del estudio se debe tener en cuenta que setratade un estudio con pocospacientes Aproximadamente el $20 \%$ de los pacientes que habían aceptado participar no ausencia de HPV-AR al momento del estudio. concurrieron a la realización del estudio. Si bien este punto no fue explorado, existen varias explicaciones posibles. Es importante considerar que, a diferencia del cáncer cervical, el cáncer anal no está instalado como problemática entre ocurre con el conocimiento de la existencia de prácticas de tamizaje. Por otra parte, los pacientes eran derivados por sus médicos en forma no sistemática, por lo cual aquellos con antecedente de patología podrían tener más posibilidad de ser enviados al estudio o de aceptar participar Por el número acotado de pacientes, el anális estadístico tambien limitado, no pudiéndose completar el análisis multivariado planeado.

Si se consideran los genotipos presentes en la vacuna contra el HPV cuadrivalente (que abarca los genotipos de bajo riesgo oncogénico HPV-6, HPV-11 y de alto riesgo oncogénico, HPV16 y HPV-18), disponible en nuestro país, se encontró HPV 6 en 20/43, HPV-11 en 9/43, HPV-16 en 22/43 y HPV-18 en 11/43. Seis pacientes no tenían ninguno de los cuatro. Si se extiende este análisis a la vacuna contra el HPV nonavalente (que amplía la cobertura a cinco genotipos más de alto riesgo; aún no disponible en nuestro país) se detectó $\mathrm{HPV}-31$ 作, y HPV-58 en $10 / 43$.

Este es uno de los primeros trabajos en estudiar tanto la citología como la presencia de HPV en nuestro país en la mayoría de los médicos y de los pacientes. Lo mis

HSH HIV+, mostrando una elevada frecuencia de HPV-AR de lesiones. En nuestro país se recomienda que todas las personas HIV+ de 11 a 26 años de edad se vacunen contra virus del papiloma humano (31). La nuevas guías de la sociedad europea extienden la edad de vacunación hasta los 40 años para el caso de los HSH (32)

Se planea continuar estudiando esta población, organizando una cohorte con un mayor número de participantes y con seguimiento longitudinal. Si bien no se ha recolectado la información en forma sistemática, algunas de las dificultades observadas en este estudio fueron la necesidad de más de una consulta médica y dificultades en coordinar los horarios de los participantes con los profesionales. Se espera adecuar os recursos para evitar estas dificultades.

Se trata de una población de alto riesgo para la presencia de lesiones anales y HPV-AR, por lo que es necesario promover y facilitar el tamizaje de cáncer anal en esta población así como evaluar estrategias de vacunación y promoción de sexo seguro.

\section{Reconocimientos}

División de Infectología del Hospital Juan A. Fernández.

Laboratorio del Hospital Juan A. Fernández.

\section{Bibliografía}

1. Crosbie, E.J., Einstein, M.H., Franceschi, S. and Kitchener, H.C., Human papillomavirus and cervical cancer. Lancet 2013. 382(9895): p. 889-99

2. Tommasino, M., The human papillomavirus family and its role in carcinogenesis. Semin Cancer Biol, 2013.

3. De Vuyst, H., Clifford, G.M. Nascimento, M.C., Madeleine M.M. and Franceschi, S., Prevalence and type distribution of human papillomavirus in carcinoma and intraepithelial neoplasia of the vulva, vagina and anus: a meta-analysis. Int J Cancer, 2009. 124(7): p. 1626-36.
4. Forman, D., de Martel, C., Lacey, C.J., Soerjomataram, I., Lortet-Tieulent, J., Bruni, L., et al., Global burden of human papillomavirus and related diseases. Vaccine, 2012. 30 Suppl 5: p. F12-23.

5. National Cancer Institute Surveillance, E.a.E.R.P. SEER Stat Fact Sheet. 19-Sep-2017]; Available from: https:// seer.cancer.gov/statfacts/htmm3/anus.htmm3.

6. Silverberg, M.J., Lau, B., Achenbach, C.J., Jing, Y., Althoff, K.N., D'Souza, G., et al., Cumulative Incidence of Cancer Among Persons With HIV in North America: A Cohort Study. Ann Intern Med, 2015. 163(7): p. 507-18. 
7. Patel, P., Hanson, D.L., Sullivan, P.S., Novak, R.M., Moorman, A.C., Tong, T.C., et al., Incidence of types of cancer among HIV-infected persons compared with the cancral population in the United States, 1992-2003. An Intern Med, 2008. 148(10): p. 728-36

8. Silverberg, M.J., Lau, B., Justice, A.C., Engels, E., Gill, M.J Goedert, J.J., et al., Risk of anal cancer in HIV-infected and HIV-uninfected individuals in North America. Clin Infect Dis, 2012. 54(7): p. 1026-34.

9. Brickman, C. and Palefsky, J.M., Human Papillomavirus in the HIV-Infected Host: Epidemiology and Pat in the Antiretroviral Era. Curr HIV/AIDS Rep, 2015. 12(1): p. 6-15.

10. D'Souza, G., Wiley, D.J., Li, X., Chmiel, J.S., Margolick, J.B. Cranston, R.D., et al., Incidence and epidemiology of anal cancer in the multicenter AIDS cohort study. J Acquir Immune Defic Syndr, 2008. 48(4): p. 491-9.

11. Crum-Cianflone, N.F., Hullsiek, K.H., Marconi, V.C. Ganesan, A., Weintrob, A., Barthel, R.V., et al., Anal cancers among HIV-infected persons: HAART is not slowing rising incidence. Aids, 2010. 24(4): p. 535-43.

12. Denny, L.A., Franceschi, S., de Sanjose, S., Heard, I, Moscicki, A.B. and Palefsky, J., Human papillomavirus, humanimmunodeficiencyvirus and immunosuppression. Vaccine, 2012. 30 Suppl 5: p. F168-74

13. Videla, S., Darwich, L., Canadas, M.P., Coll, J., Pinol M., Garcia-Cuyas, F., et al., Natural history of human papillomavirus infections involving anal, penile, and ora sites among HIV-positive men. Sex Transm Dis, 2013. 40(1): p. 3-10.

14. Wasserman, P., Rubin, D.S. and Turett, G., Review: Ana Intraepithelial Neoplasia in HIV-Infected Men Who Have Sex with Men: Is Screening and Treatment Justified? AIDS Patient Care STDS, 2017. 31(6): p. 245-253.

15. Berry, J.M., Jay, N., Cranston, R.D., Darragh, T.M., Holly, E.A., Welton, M.L., et al., Progression of anal high-grade squamous intraepithelial lesions to invasive anal cancer among HIV-infected men who have sex with men. Int $J$ Cancer, 2014. 134(5): p. 1147-55.

16. Pando, M.A., Balan, I.C., Marone, R., Dolezal, C., Leu, C.S. Squiquera, L., et al., HIV and other sexually transmitted infections among men who have sex with men recruited by RDS in Buenos Aires, Argentina: high HIV and HPV infection. PLoS One, 2012. 7(6): p. e39834.

17. D. Forman, F.B., D.H. Brewster, C. Gombe Mbalawa, B. Kohler, M. Piñeros, E. Steliarova-Foucher, R. Swaminathan, J. Ferlay Cancer Incidence in Five Continents Vol. X. 2014. X

18. Solomon Diane, N.R., The Bethesda System for Reporting Cervical Cytology Definitions, Criteria, and Explanatory
Notes 2004: Springer Science \& Business Media.

González, F., Bouzas, M.B., Biología molecular, genotipificación del HPV y sus implicancias en la práctica clínica , in HPV Guía de Manejo Multidisciplinario, L. Svidler López, Presencia, G, Sidra, G, Pastore, R. , Editor. 2016: Buenos Aires.

20. Machalek, D.A., Poynten, M., Jin, F., Fairley, C.K. Farnsworth, A., Garland, S.M., et al., Anal human papillomavirus infection and associated neoplastic lesions in men who have sex with men: a systematic

21. Torres, M., Gonzalez, C., del Romero, J., Viciana, P., Ocampo, A., Rodriguez-Fortunez, P., et al., Anal human papillomavirus genotype distribution in HIV-infected men who have sex with men by geographical origin, age, and cytological status in a Spanish cohort. J Clin Microbiol, 2013. 51(11): p. 3512-20

22. Taylor, S., Bunge, E., Bakker, M. and Castellsague, X., The incidence, clearance and persistence of non-cervical human papillomavirus infections: a systematic review of the literature. BMC Infect Dis, 2016. 16: p. 293.

23. Mendez-Martinez, R., Rivera-Martinez, N.E., CrabtreeRamirez, B., Sierra-Madero, J.G., Caro-Vega, Y., Galvan, S.C. et al., Multiple human papillomavirus infections are highly prevalent in the anal canal of human immunodeficiency virus-positive men who have sex with men. BMC Infect Dis, 2014. 14: p. 671.

24. van Aar, F., Mooii, S.H., van der Sande, M.A., Speksnijder A.G., Stolte, I.G., Meijer, C.J., et al., Anal and penile highrisk human papillomavirus prevalence in HIV-negative and HIV-infected MSM. AIDS, 2013. 27(18): p. 2921-31.

25. Nowak, R.G., Gravitt, P.E., He, X., Ketende, S., Dauda, W. Omuh, H., et al., Prevalence of Anal High-Risk Human Papillomavirus Infections Among HIV-Positive and HIVNegative Men Who Have Sex With Men in Nigeria. Sex Transm Dis, 2016. 43(4): p. 243-8.

26. Stier,E.A., Sebring, M.C., Mendez,A.E.,Ba, F.S., Trimble, D.D. and Chiao, E.Y., Prevalence of anal human papillomavirus infection and anal HPV-related disorders in women: a systematic review. Am J Obstet Gynecol, 2015.

27. Bertisch, B., Franceschi, S., Lise, M., Vernazza, P., Keiser, O., Schoni-Affolter, F., et al., Risk factors for anal cancer in persons infected with HIV: a nested case-control study in the Swiss HIV Cohort Study. Am J Epidemiol, 2013. 178(6): p. 877-84.

28. Conley, L., Bush, T., Darragh, T.M., Palefsky, J.M., Unger, E.R., Patel, P., et al., Factors associated with prevalent abnormal anal cytology in a large cohort of HIV-infected adults in the United States. J Infect Dis, 2010. 202(10): p.
1567-76.

29. Lam, J.U., Rebolj, M., Dugue, P.A., Bonde, J., von EulerChelpin, M. and Lynge, E., Condom use in prevention of Human Papillom systematic review of longitudinal studies. J Med Screen 2014. 21(1): p. 38-50

30. 30. Repp, K.K., Nielson, C.M., Fu, R., Schafer, S., LazcanoPonce, E., Salmeron, J., et al., Male human papillomavirus prevalence and association with condom use in Brazi Mexico, and the United States. J Infect Dis, 2012. 205(8): p. 1287-93.

31. 31. ProNaCei, M.d.S., Vacunación en Huéspedes Especiales Lineamientos Técnicos Argentina 2014. 2014.

32. 32. European AIDS Clinical Society. EACS Guidelines for treatment of HIV- positive adults. 2017 21-nov2017l: Avaiable from: http://www.eacsociety.org/files/ guidelines_9.0-english.pdf.
HIGH RISK HPV INFECTION AND ANAL INTRAEPITHELIAL LESIONS IN HIV POSITIVE MEN WHO HAVE SEX WITH MEN

Introduction: Anal cancer, associated with the infection with high risk Human Papillomavirus (HR-HPV), is very frequent among HIV+ men who have sex with men (MSM).

Objective: To evaluate the frequency of HR-HPV infection presence of HPV genotypes and HPV-associated lesions and associated factors.

Methods: Study in HIV+ MSM (September 2012- March 2014, Hospital Fernández). Demographical, HIV, HPV and sexual behaviour information was collected. Cytology, HRHPV detection (HC2 High-Risk HPV DNA, Digene ${ }^{\circledR}$ ) and genotyping was performed on samples positive for HR-HPV (Inno Lipa®, Fujirebio). All patients signed informed consent. Treatment was provided according to results.

Results: Fifty-seven patients completed the study. Median age was 40 years old (interquartile range [IQR]: 29-45); median CD4 cell count: 444 cels/mm3 (IQR: 345-568); 77\% were under ARV treatment, $68 \%$ with undetectable viral load. Cytology results: $24 \%$ negative, $54 \%$ low grade intraepithelial lesion, $20 \%$ high grade intraepithelial lesion, $2 \%$ ASCUS. Eighty percent were HR-HPV+. Patients with HR-HPV $(p=0,006)$ and diagnosis of intraepithelial lesion had more frequent CD4 $<500$ cels $/ \mathrm{mm} 3(p=0,030)$. Patients diagnosed with HR-HPV had a higher frequency of detectable viral load $(p=0,020$, prueba de Fisher). The percentage of patients with consistent condom use was higher among patients without cytological lesion $(\mathrm{p}=0,026)$.Most frequent high risk genotypes: HPV-16 (51\%), HPV-31 (44\%) and HPV-51 (40\%); low risk genotypes HPV-6 (47\%) and HPV-44 (35\%).

Conclusions: There was high frequency of cytological lesions $(76 \%)$ and $\mathrm{HR}$-HPV $(80 \%)$. It is necessary to promote prevention strategies in this population including screening, vaccine and safe sex promotion

Key words: HIV, anal intraepithelial lesion, HPV, anal cytology, anal cancer screening, men who have sex with men. 